\title{
Smoothing a Program Soundly and Robustly
}

\author{
Swarat Chaudhuri ${ }^{1}$ and Armando Solar-Lezama ${ }^{2}$ \\ ${ }^{1}$ Rice University \\ 2 MIT
}

\begin{abstract}
We study the foundations of smooth interpretation, a recentlyproposed program approximation scheme that facilitates the use of local numerical search techniques (e.g., gradient descent) in program analysis and synthesis. While the popular techniques for local optimization works well only on relatively smooth functions, functions encoded by real-world programs are infested with discontinuities and local irregular features. Smooth interpretation attenuates such features by taking the convolution of the program with a Gaussian function, effectively replacing discontinuous switches in the program by continuous transitions. In doing so, it extends to programs the notion of Gaussian smoothing, a popular signal-processing technique used to filter noise and discontinuities from signals.

Exact Gaussian smoothing of programs is undecidable, so algorithmic implementations of smooth interpretation must necessarily be approximate. In this paper, we characterize the approximations carried out by such algorithms. First, we identify three correctness propertiessoundness, robustness, and $\beta$-robustness - that an approximate smooth interpreter should satisfy. In particular, a smooth interpreter is sound if it computes an abstraction of a program's "smoothed" semantics, and robust if it has arbitrary-order derivatives in the input variables at every point in its input space. Second, we describe the design of an approximate smooth interpreter that provably satisfies these properties. The interpreter combines program abstraction using a new domain with symbolic calculation of convolution.
\end{abstract}

\section{Introduction}

Smooth interpretation [4 is a recently-proposed program transformation permitting more effective use of numerical optimization in automated reasoning about programs. Many problems in program analysis and synthesis can be framed as optimization questions - examples include finding program parameters so that the resultant program behavior is as close as possible to a specification [4, or the generation of tests that maximize the number of times a certain operation is executed [1]. But rarely are such problems solvable using off-the-shelf numerical optimization engines. This is because search spaces arising in real-world programs are rife with discontinuities - on such spaces, local search algorithms like gradient descent or Newton iteration find themselves unable to converge on a good solution. It is this predicament that smooth interpretation tries to resolve.

\footnotetext{
* This work was supported by NSF CAREER Award \#0953507 and the MIT CSAIL.
}

G. Gopalakrishnan and S. Qadeer (Eds.): CAV 2011, LNCS 6806, pp. 277 2922011.

(C) Springer-Verlag Berlin Heidelberg 2011 
To aid numerical search over the input space of a program $P$, smooth interpretation transforms $P$ into a smooth mathematical function. For example, if the semantics of $P$ (viewed as a function $P\left(x_{1}, x_{2}\right)$ of inputs $x_{1}$ and $\left.x_{2}\right)$ is the discontinuous map in Fig. 1 (a), the "smoothed" version of $P$ will typically have semantics as in Fig. 1 1 (b). More precisely, smooth interpretation extends to programs the notion of Gaussian smoothing [12, an elementary signal-processing technique for filtering out noise and discontinuities from ill-behaved real-world signals. To perform Gaussian smoothing of a signal, one takes the convolution of the signal with a Gaussian function. Likewise, to smooth a program, we take the convolution of the denotational semantics of $P$ with a Gaussian function.

The smoothing transformation is parameterized by a value $\beta$ which controls the degree of smoothing. Numerical search algorithms will converge faster and find better local minima when $\beta$ is high and the function is very smooth, but a bigger $\beta$ also introduces imprecision, as the minima that is found when using a big $\beta$ may be far from the real minima. The numerical optimization algorithm from [4] addresses this by starting with a high value of $\beta$ then reducing it every time a minima is found. When $\beta$ is reduced, the location of the last minima is used as a starting point for a new round of numerical search.

In our previous work, we showed the effectiveness of this approach for the problem of embedded controller synthesis. Specifically, we showed that the algorithm defined above could find optimal parameters for interesting controllers where simple numerical search would fail. But the benefits of the technique are not limited to parameter syn-

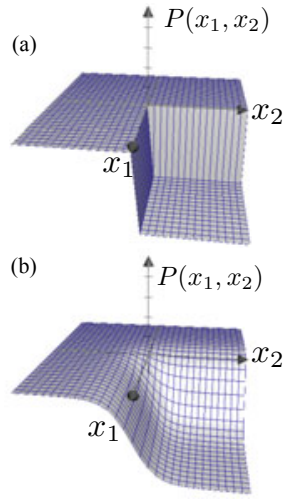

Fig. 1. (a) A discontinuous program. (b) After smoothing. thesis; smooth interpretation constitutes a wholly new form of program approximation, and is likely to have broad impact by opening the door to a wide array of applications of numerical optimization in program analysis.

Smooth interpretation exhibits many parallels with program abstraction, but it also introduces some new and important concerns. The goal of this paper is to understand these concerns by analyzing the foundations of smooth interpretation. In particular, we seek to characterize the approximations that must be made by algorithmic implementations of program smoothing given that computing the exact Gaussian convolution of an arbitrary program is undecidable.

Our concrete contributions are the following:

1. We identify three correctness properties that an algorithmic (and therefore approximate) implementation of smooth interpretation should ideally satisfy: soundness, robustness, and $\beta$-robustness.

While the notion of soundness here is related to the corresponding notion in program abstraction, the two notions are semantically quite different: a sound smooth interpreter computes an abstraction of a "smoothed" semantics of programs. As for robustness, this property states that the function computed by an approximate smooth interpreter has a linearly bounded 
derivative at every point in its input space-i.e., that even at the points in the input space where $P$ is discontinuous or non-differentiable, the smoothed version of $P$ is only as "steep" as a quadratic function. This property allows gradient-based optimization techniques to be applied to the programindeed, many widely used algorithms for gradient-based nonlinear optimization 107] are known to perform best under such a guarantee.

As for $\beta$-robustness, this property demands that the output of the smooth interpreter has a small partial derivative in $\beta$. The property is important to the success of the iterative algorithm described above, which relies on repeated numerical search with progressively smaller values of $\beta$. Without $\beta$-robustness, it would be possible for the approximation to change dramatically with small changes to $\beta$, making the algorithm impractical.

2. We give a concrete design for an approximate smooth interpreter (called $\left.\operatorname{Smooth}_{P}(\mathbf{x}, \beta)\right)$ that satisfies the above properties. The framework combines symbolic computation of convolution with abstract interpretation: when asked to smooth a program $P, \operatorname{Smooth}_{P}(\mathbf{x}, \beta)$ uses an abstract interpreter to approximate $P$ by a pair of simpler programs $P_{\text {inf }}$ and $P_{\text {sup }}$, then performs a symbolic computation on these approximations. The result of this computation is taken as the semantics of the smoothed version of $P$.

The soundness of our method relies on the insight that Gaussian convolution is a monotone map on the pointwise partial order of vector functions. We establish robustness and $\beta$-robustness under a weak assumption about $\beta$, by bounding the derivative of a convolution. Thus, the techniques used to prove our analysis correct are very different from those in traditional program analysis. Also, so far as we know, the abstract domain used in our construction is new to the program analysis literature.

The paper is structured as follows. In Sec. 2] we recapitulate the elements of smooth interpretation and set up the programming language machinery needed for our subsequent development. In Sec. 3, we introduce our correctness requirements for smoothing; in Sec. 4, we present our framework for smooth interpretation. Sec. [5] studies the properties of interpreters derived from this framework. Our discussion of related work, as well as our conclusions, appear in Sec. 6.

\section{Smooth Interpretation}

We begin by fixing, for the rest of the paper, a simple language of programs. Our programs are written in a flow-graph syntax [8, and maintain their state in $k$ real-valued variables named $\mathrm{x}_{1}$ through $\mathrm{x}_{\mathrm{k}}$.

Formally, let $R e$ denote the set of linear arithmetic expressions over $\mathrm{x}_{1}, \ldots, \mathrm{x}_{\mathrm{k}}$, encoding linear transformations of the type $\mathbb{R}^{k} \rightarrow \mathbb{R}^{k}$. Also, let $B e$ the set of boolean expressions of the form $Q>0$ or $Q \geq 0$, where $Q \in \mathbb{R}^{k} \rightarrow \mathbb{R}$. A program $P$ in our language is a directed graph. Nodes of this graph can be of five types:

- An entry node has one outgoing edge and no incoming edge, and an exit node has one incoming edge and no outgoing edge. A program has a single entry node and a single exit node. 
- An assignment node has a single incoming edge and single outgoing edge. Each assignment node $u$ is labeled with an expression $E \in R e$. Intuitively, this expression is the r-value of the assignment.

- A test node $u$ has one incoming edge and two outgoing edges (known as the true and false-edges), and is labeled by a boolean expression $\operatorname{Test}(u) \in B e$. Intuitively, $u$ is a conditional branch.

- A junction node has a single outgoing edge and two incoming edges.

For example, Fig. 2 depicts a simple program over a single variable.

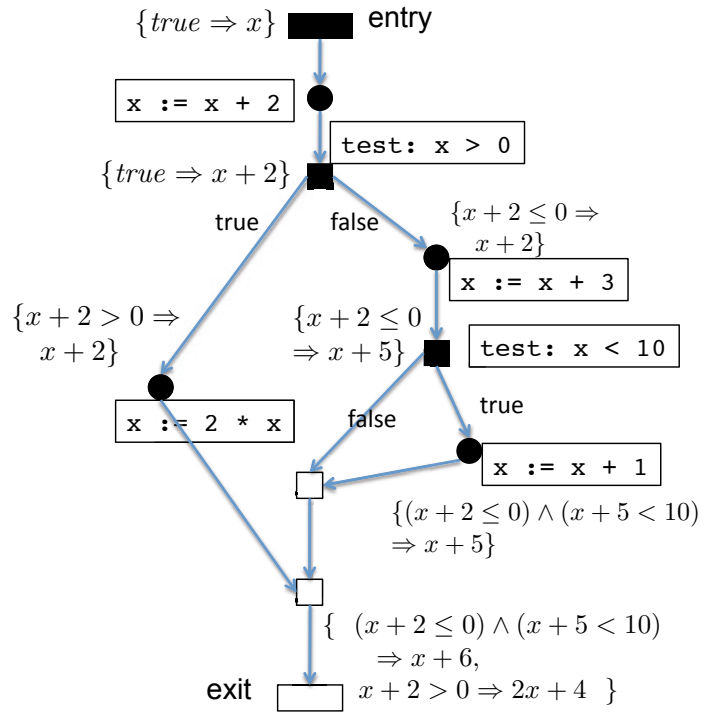

Fig. 2. A program and its collecting semantics
Semantics. The intuitive operational semantics of $P$ is that it starts executing at its entry node, taking transitions along the edges, and terminates at the exit node. For our subsequent development, however, a denotational semantics as well as an abstract-interpretationstyle collecting semantics are more appropriate than an operational one. Now we define these semantics.

Let a state of $P$ be a vector $\mathbf{x}=\left\langle x_{1}, \ldots, x_{k}\right\rangle \in \mathbb{R}^{k}$, where each $x_{i}$ captures the value of the variable $x_{i}$. For each arithmetic expression $E \in R e$, the denotational semantics $\llbracket E \rrbracket(\mathbf{x}): \mathbb{R}^{k} \rightarrow \mathbb{R}^{k}$ produces the value of $E$ at the state $\mathbf{x}$.

For each $B \in B e$, the denotation function $\llbracket B \rrbracket$ produces $\llbracket B \rrbracket(\mathbf{x})=0$ if $B$ is false at the state $\mathbf{x}$, and 1 otherwise.

To define the semantics of $P$, we need some more machinery. Let a guarded linear expression be an expression of the form

\section{if $B$ then $F$ else $\mathbf{0}$,}

where $B$ is a conjunction of linear inequalities over the variables $x_{1}, \ldots, x_{k}$, and $F$ is a linear arithmetic expression. We abbreviate the above expression by the

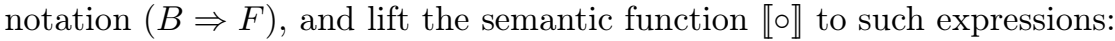

$$
\llbracket B \Rightarrow F \rrbracket(\mathbf{x})=\text { if } \llbracket B \rrbracket(\mathbf{x}) \text { then } \llbracket F \rrbracket(\mathbf{x}) \text { else } \mathbf{0}=\llbracket B \rrbracket(\mathbf{x}) \cdot \llbracket F \rrbracket(\mathbf{x}) .
$$

The collecting semantics of $P$ is given by a map $\Psi_{P}$ that associates with each node $u$ of $P$ a set $\Psi_{P}(u)$ of guarded linear expressions. Intuitively, each such expression captures the computation carried out along a path in $P$ ending at $u$. As the program $P$ can have "loops," $\Psi_{P}(u)$ is potentially infinite. 
In more detail, we define the sets $\Psi_{P}(u)$ so that they form the least fixpoint of a monotone map. For each node $u$ of $P, \Psi_{P}(u)$ is the least set of guarded linear expressions satisfying the following conditions:

- If $u$ is the entry node and its out-edge goes to $v$, then $\{($ true $\Rightarrow \mathbf{x})\} \subseteq \Psi_{P}(v)$.

- Suppose $u$ is an assignment node labeled by $E$ and its outgoing edge leads to $v$. Let $\circ$ be the usual composition operator over expressions. Then for all $(B \Rightarrow F) \in \Psi_{P}(u)$, the expression $B \Rightarrow(E \circ F)$ is in $\Psi_{P}(v)$.

- Suppose $u$ is a branch node, and let $v_{t}$ and $v_{f}$ respectively be the targets of the true- and false-edges out of it. Let $Q_{t}=\operatorname{Test}(u) \circ F$ and $Q_{f}=$ $(\neg$ Test $(u)) \circ F$; then, for all $(B \Rightarrow F) \in \Psi_{P}(u)$, we have

$$
\left(B \wedge Q_{t}\right) \Rightarrow F \in \Psi_{P}\left(v_{t}\right) \quad\left(B \wedge Q_{f}\right) \Rightarrow F \in \Psi_{P}\left(v_{f}\right) .
$$

- If $u$ is a junction node and its outgoing edge leads to $v$, then $\Psi_{P}(u) \subseteq \Psi_{P}(v)$.

For example, consider the program in Fig. 2, Most nodes $u$ of this program are labeled with $\Psi_{P}(u)$. We note that one of the three control flow paths to the exit node $e x$ is infeasible; as a result $\Psi_{P}(e x)$ has two formulas rather than three.

The denotational semantics $\llbracket P \rrbracket$ of $P$ is now defined using the above collecting semantics. Let $e x$ be the exit node of $P$. We define:

$$
\llbracket P \rrbracket(\mathbf{x})=\sum_{(B \Rightarrow F) \in \Psi_{P}(e x)} \llbracket B \rrbracket(\mathbf{x}) \cdot \llbracket F \rrbracket(\mathbf{x}) .
$$

Intuitively, for any $\mathbf{x} \in \mathbb{R}^{k}, \llbracket P \rrbracket(\mathbf{x})$ is the output of $P$ on input $\mathbf{x}$.

Smoothed semantics. Now we recall the definition [4 of the smoothed semantics of programs, which is the semantics that smooth interpretation seeks to compute. To avoid confusion, the previously defined semantics is from now on known as the crisp semantics.

Let $\beta>0$ be a real-valued smoothing parameter, and let $\mathcal{N}(\mathbf{x}, \beta)$ be the joint density function of $k$ independent normal variables, each with mean 0 and standard deviation $\beta$. In more detail, letting $\mathbf{x}=\left\langle x_{1}, \ldots, x_{k}\right\rangle$ as before, $\mathcal{N}(\mathbf{x}, \beta)$ is given by $\mathcal{N}(\mathbf{x}, \beta)=\frac{1}{\left(2 \pi \beta^{2}\right)^{k / 2}} e^{-\frac{\sum_{i=1}^{k} x_{i}^{2}}{2 \beta^{2}}}=\prod_{i=0 . . k} \mathcal{N}\left(x_{i}\right)$.

The smoothed semantics $\overline{\llbracket P \rrbracket}_{\beta}: \mathbb{R}^{k} \rightarrow \mathbb{R}^{k}$ of a program $P$ with respect to $\beta$ is obtained by taking the convolution of $\mathcal{N}$ and the crisp semantics of $P$ as shown be the following equation.

$$
\begin{aligned}
\overline{\llbracket P \rrbracket}_{\beta}(\mathbf{x}) & =\int_{\mathbf{r} \in \mathbb{R}^{k}} \llbracket P \rrbracket(\mathbf{r}) \mathcal{N}(\mathbf{x}-\mathbf{r}, \beta) d \mathbf{r} \\
& =\sum_{(B \Rightarrow F) \in \Psi_{P}(e x)} \int_{\mathbf{r} \in \mathbb{R}^{k}} \llbracket B \Rightarrow F \rrbracket(\mathbf{r}) \mathcal{N}(\mathbf{x}-\mathbf{r}, \beta) d \mathbf{r} .
\end{aligned}
$$

As before, ex refers to the exit node of $P$. Note that because convolution is a commutative operator, we have the property

$$
\overline{\llbracket P \rrbracket}_{\beta}(\mathbf{x})=\int_{\mathbf{r} \in \mathbb{R}^{k}} \llbracket P \rrbracket(\mathbf{x}-\mathbf{r}) \mathcal{N}(\mathbf{r}, \beta) d \mathbf{r} .
$$


When $\beta$ is clear from context, we denote $\overline{\llbracket P \rrbracket}_{\beta}$ by $\overline{\llbracket P \rrbracket}$, and $\mathcal{N}(\mathbf{x}, \beta)$ by $\mathcal{N}(\mathbf{x})$. One of the properties of smoothing is that even if $\llbracket P \rrbracket$ is highly discontinuous, $\overline{\llbracket P \rrbracket}$ is a smooth mathematical function that has all its derivatives defined at every point in $\mathbb{R}^{k}$. The smoothing parameter $\beta$ can be used to control the extent to which $\llbracket P \rrbracket$ is smoothed by the above operation - the higher the value of $\beta$, the greater the extent of smoothing.

Example 1. Consider a program $P$ over one variable $x$ such that $\llbracket P \rrbracket(x)=$ if $x>$ $a$ then 1 else 0 , where $a \in \mathbb{R}$. Let erf be the Gauss error function. We have

$$
\begin{gathered}
\overline{\llbracket P \rrbracket}(x)=\int_{-\infty}^{\infty} \llbracket P \rrbracket(y) \mathcal{N}(x-y) d y=0+\int_{a}^{\infty} \mathcal{N}(x-y) d y \\
=\int_{0}^{\infty} \frac{1}{\sqrt{2 \pi} \beta} e^{-(y-x+a)^{2} / 2 \beta^{2}} d y=\frac{1+\operatorname{erf}\left(\frac{x-a}{\sqrt{2} \beta}\right)}{2}
\end{gathered}
$$

Figure 3 -(a) plots the crisp semantics $\llbracket P \rrbracket$ of $P$ with $a=2$, as well as $\overline{\llbracket P \rrbracket}$ for $\beta=0.5$ and $\beta=3$. While $\llbracket P \rrbracket$ has a discontinuous "step," $\overline{\llbracket P \rrbracket}$ is a smooth S-shaped curve, or a sigmoid. Note that as we decrease the "tuning knob" $\beta$, the sigmoid $\overline{\llbracket P \rrbracket}$ becomes steeper and steeper, and at the limit, approaches $\llbracket P \rrbracket$.

Now consider the program $P^{\prime}$ such that $\llbracket P^{\prime} \rrbracket(x)=$ if $a<x<c$ then 1 else 0 , where $a, c \in \mathbb{R}$ and $a<c$. The "bump-shaped" functions obtained by smoothing $P^{\prime}$ are plotted in Figure 3 (b) (here $a=-5, c=5$, and $\beta$ has two values 0.5 and 2). Note how the discontinuities are smoothed.

Now we consider an even more interesting program, one that is key to the main results of this paper. Consider $\llbracket P^{\prime \prime} \rrbracket(x)=\llbracket B \Rightarrow F \rrbracket(x)$, where $B$ is the boolean expression $a<x<b$ for constants $a, b$, and $F(x)=\alpha \cdot x+\gamma$ for constants $\alpha, \gamma$. In this case, the smoothed semantics of $P^{\prime \prime}$ can be evaluated symbolically as follows:

$$
\begin{aligned}
\overline{\llbracket P^{\prime \prime} \rrbracket}(x) & =\int_{a}^{b}(\alpha y+\gamma) \mathcal{N}(x-y) d y=\alpha \int_{a}^{b} y \mathcal{N}(x-y) d y+\gamma \int_{a}^{b} \mathcal{N}(x-y) d y \\
& =\frac{(\alpha x+\gamma) \cdot\left(\operatorname{erf}\left(\frac{b-x}{\sqrt{2} \beta}\right)-\operatorname{erf}\left(\frac{a-x}{\sqrt{2} \beta}\right)\right)}{2}+\frac{\beta \alpha\left(e^{-(a-x)^{2} / 2 \beta^{2}}-e^{-(b-x)^{2} / 2 \beta^{2}}\right)}{\sqrt{2 \pi}} .
\end{aligned}
$$
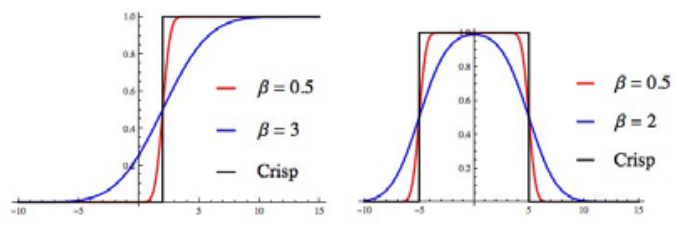

Fig. 3. (a) A sigmoid (b) A bump
Smooth interpretation. We use the phrase "smooth interpreter of a program $P$ " to refer to an algorithm that can execute $P$ according to the smoothed semantics ${\overline{\llbracket \circ \rrbracket_{\beta}}}_{\beta}$. The primary application of smooth interpretation is to enable the use of numerical search for parameter optimization problems. For example, suppose our goal is to compute an input $\mathbf{x}$ for which $\llbracket P \rrbracket(\mathbf{x})$ is minimal. For a vast number of real-world programs, discontinuities in $\llbracket P \rrbracket$ would preclude the use of local numerical methods in this minimization problem. By eliminating such discontinuities, smooth interpretation can make numerical search practical. 
In a way, smooth interpretation is to numerical optimization what abstraction is to model checking: a mechanism to approximate challenging local transitions in a program-derived search space. But the smoothed semantics that we use is very different from the collecting semantics used in abstraction. Rather, the smoothed semantics of $P$ can be seen to be the expectation of a probabilistic semantics of $P$.

Consider an input $\mathbf{x} \in \mathbb{R}^{k}$ of $P$, and suppose that, before executing $P$, we randomly perturb $\mathbf{x}$ following a $k$-D normal distribution with independent components and standard deviation $\beta$. Thus, the input of $P$ is now a random variable $X$ following a normal distribution $\mathcal{N}$ with mean $\mathbf{x}$ and similar shape as the one used in the perturbation. Now let us execute $P$ on $\mathrm{X}$ with crisp semantics. Consider the expectation of the output $\llbracket P \rrbracket(\mathbf{X}): \mathbf{E x p}[\llbracket P \rrbracket(\mathbf{X})]=$ $\int_{-\infty}^{\infty} \llbracket P \rrbracket(\mathbf{r}) \mathcal{N}(\mathbf{x}-\mathbf{r}) d \mathbf{r}=\overline{\llbracket P \rrbracket}(\mathbf{x})$. Here $\overline{\llbracket P \rrbracket}$ is computed using the Gaussian $\mathcal{N}$. In other words, the smoothed semantics of $P$ is the expected crisp semantics of $P$ under normally distributed perturbations to the program inputs.

Now that we have defined how a smooth interpreter must behave, consider the question of how to algorithmically implement such an interpreter. On any input $\mathbf{x}$, an idealized smooth interpreter for $P$ must compute $\overline{\llbracket P \rrbracket}_{\beta}(\mathbf{x})$ - in other words, integrate the semantics of $P$ over a real space. This problem is of course undecidable in general; therefore, any algorithmic implementation of a smooth interpreter must necessarily be approximate. But when do we judge such an approximation to be "correct"? Now we proceed to answer this question.

\section{Approximate Smooth Interpreters and their Correctness}

In this section, we define three correctness properties for algorithmic implementations of smooth interpretation: soundness, robustness, and $\beta$-robustness. While an algorithm for smooth interpretation of a program must necessarily be approximate, these desiderata impose limits on the approximations that it makes.

Formally, we let an approximate smooth interpreter $\operatorname{Smooth}_{P}(\mathbf{x}, \beta)$ for $P$ be an algorithm with two inputs: an input $\mathbf{x} \in \mathbb{R}^{k}$ and a smoothing parameter $\beta \in \mathbb{R}^{+}$. Given these, $\operatorname{Smooth}_{P}$ returns a symbolic representation of a set $\mathbf{Y} \subseteq$ $\mathbb{R}^{k}$. To avoid notation-heavy analytic machinery, we restrict the sets returned by Smooth $_{P}$ to be intervals in $\mathbb{R}^{k}$. Recall that such an interval is a Cartesian product $\left\langle\left[l_{1}, u_{1}\right], \ldots,\left[l_{k}, u_{k}\right]\right\rangle$ of intervals over $\mathbb{R}$; the interval can also be represented more conveniently as a pair of vectors $\left[\left\langle l_{1}, \ldots, l_{k}\right\rangle,\left\langle u_{1}, \ldots, u_{k}\right\rangle\right]$; from now on, we denote the set of all such intervals by $\mathbf{I}$.

Soundness. Just as a traditional static analysis of $P$ is sound if it computes an abstraction of the crisp semantics of $P$, an approximate smooth interpreter is sound if it abstracts the smoothed semantics of $P$. In other words, the interval returned by $\operatorname{Smooth}_{P}$ on any input $\mathbf{x}$ bounds the output of the idealized smoothed version of $P$ on $\mathbf{x}$. We define:

Definition 1 (Soundness). An approximate smooth interpreter Smooth $_{P}$ : $\mathbb{R}^{k} \times \mathbb{R}^{+} \rightarrow \mathbf{I}$ is sound iff for all $\mathbf{x} \in \mathbb{R}^{k}, \overline{\llbracket P \rrbracket}_{\beta}(\mathbf{x}) \in \operatorname{Smooth}_{P}(\mathbf{x}, \beta)$. 
Robustness. A second requirement, critical for smooth interpreters but less relevant in abstract interpretation, is robustness. This property asserts that for all $\mathbf{x}$ and $\beta, \operatorname{Smooth}_{P}(\mathbf{x}, \beta)$ has derivatives of all orders, and that further, its partial derivative with respect to the component scalars of $\mathbf{x}$ is small-i.e., bounded by a function linear in $\mathbf{x}$ and $\beta$.

The first of the two requirements above simply asserts that $\operatorname{Smooth}_{P}(\mathbf{x}, \beta)$ computes a smooth function, in spite of all the approximations carried out for computability. The rationale behind the second requirement is apparent when we consider smooth interpretation in the broader context of local numerical optimization of programs. A large number of numerical search routines work by sampling the input space under the expectation that the derivative around each sample point is well defined. Our requirement guarantees this.

In fact, by putting a linear bound on the derivative of $\operatorname{Smooth}_{P}$, we give a stronger guarantee: the absence of regions of extremely steep descent that can lead to numerical instability. Indeed, robustness implies that even at the points in the input space where $P$ is discontinuous, the gradient of Smooth $_{P}$ is Lipschitz-continuous - i.e., Smooth $_{P}$ is only as "steep" as a quadratic function. Many algorithms for nonlinear optimization [10/7 demand a guarantee of this sort for best performance. As we will see later, we can implement a smooth interpreter that is robust (under a weak additional assumption) even in our strong sense. Let us now define:

Definition 2 (Robustness). $\operatorname{Smooth}_{P}(\mathbf{x}, \beta)$ is robust if $\frac{\partial}{\partial x_{i}} \operatorname{Smooth}_{P}(\mathbf{x}, \beta)$ exists at all $\mathbf{x}=\left\langle x_{1}, \ldots, x_{k}\right\rangle$ and for all $i$, and there exists a linear function $K(\mathbf{x}, \beta)$ in $\mathbf{x}$ that satisfies $\left\|\frac{\partial}{\partial x_{i}} \operatorname{Smooth}_{P}(\mathbf{x}, \beta)\right\| \leq K(\mathbf{x}, \beta)$ for all $\mathbf{x}, i, \beta$.

The definition above abuses notation somewhat because, as you may recall, $\operatorname{Smooth}_{P}(\mathbf{x}, \beta)$ actually produces a Cartesian product of intervals, as opposed to a real number; so the derivative $\frac{\partial}{\partial x_{i}} \operatorname{Smooth}_{P}(\mathbf{x}, \beta)$ is actually a pair of vectors $\left[\left\langle\frac{\partial}{\partial x_{i}} l_{1}, \ldots, \frac{\partial}{\partial x_{i}} l_{k}\right\rangle,\left\langle\frac{\partial}{\partial x_{i}} u_{1}, \ldots, \frac{\partial}{\partial x_{i}} u_{k}\right\rangle\right]$. The measure for such a pair of vectors is a simple Euclidian measure that adds the squares of each of the components.

$\beta$-robustness. Another correctness property for an approximate smooth interpreter is that it produces functions that have small partial derivatives with respect to the smoothing parameter $\beta$. In more detail, the derivative $\frac{\partial \operatorname{Smooth}_{P}(\mathbf{x}, \beta)}{\partial \beta}$ must be bounded by a function linear in $\mathbf{x}$ and $\beta$. We consider this property important because of the way our numerical search algorithm from [4] uses smoothing: starting with a large $\beta$ and progressively reducing it, improving the quality of the approximation in a way akin to the abstraction-refinement loop in program verification. The property of $\beta$-robustness guarantees that the functions optimized in two successive iterations of this process are not wildly different. In other words, the optima of one iteration of the process do not become entirely suboptimal in the next iteration.

Formally, we define the property of $\beta$-robustness as follows:

Definition 3 (Robustness in $\beta$ ). $\operatorname{Smooth}_{P}(\mathbf{x}, \beta)$ is robust in $\beta$ if the partial derivative $\frac{\partial}{\partial \beta} \operatorname{Smooth}_{P}(\mathbf{x}, \beta)$ exists for all $\beta>0$, and there is a linear function $K(\mathbf{x}, \beta)$ such that for all $\mathbf{x}$ and $\beta,\left\|\frac{\partial}{\partial \beta} \operatorname{Smooth}_{P}(\mathbf{x}, \beta)\right\| \leq K(\mathbf{x}, \beta)$. 


\section{Designing a Smooth Interpreter}

Now we present a framework for approximate smooth interpretation that satisfies the correctness properties defined in the previous section. We exploit the fact that under certain restrictions on a program $P$, it is possible to build an exact smooth interpreter for $P$-i.e., an algorithm that computes the smoothed semantics $\overline{\llbracket P \rrbracket}$ exactly. The idea behind our construction is to approximate $P$ by programs for which exact smooth interpreters can be constructed.

Let us consider guarded linear expressions (defined in Sec. 2); recall that for nodes $u$ of $P, \Psi_{P}(u)$ is a possibly-infinite set of guarded linear expressions $(B \Rightarrow F)$. By Eqn. (1), computing the exact smoothed semantics of $P$ amounts to computing a sum of Gaussian convolutions of guarded linear expressions.

Unfortunately, the convolution integral of a general guarded linear expression does not have a clean symbolic solution. We overcome this problem by abstracting each such expression using an interval-guarded linear expression $B_{\text {int }} \Rightarrow F$, where $B_{\text {int }}$ is an interval in $\mathbb{R}^{k}$ obtained through a Cartesian abstraction of $B$. From an argument as in Example 1, if an expression is interval-guarded and linear, then its exact smoothed semantics can be computed in closed-form.

The above strategy alone is not enough to achieve convergence, given that $\Psi_{P}(u)$ can be infinite. Hence we use pairs of interval-guarded linear expressions of the form $\left\langle\left(B_{\text {int }} \Rightarrow F_{\text {sup }}\right),\left(B_{\text {int }} \Rightarrow F_{\text {inf }}\right)\right\rangle$ to abstract unbounded sets of linear expressions guarded by subintervals of $B_{\text {int }}$. Such a tuple is known as an intervalguarded bounding expression, and abbreviated by the notation $\left\langle B_{\text {int }}, F_{\text {sup }}, F_{\text {inf }}\right\rangle$.

To see what such an abstraction means, let us define the pointwise ordering relation $\preceq$ among functions of type $\mathbb{R}^{k} \rightarrow \mathbb{R}^{k}$ as follows: $F_{1} \preceq F_{2}$ iff for all $\mathbf{x} \in \mathbb{R}^{k}$, we have $F_{1}(\mathbf{x}) \leq F_{2}(\mathbf{x})$. We lift this function to arithmetic expressions $E$, letting $E_{1} \preceq E_{2}$ iff $\llbracket E_{1} \rrbracket \preceq \llbracket E_{2} \rrbracket$. We guarantee that if $\left\langle B_{\text {int }}, F_{\text {sup }}, F_{\text {inf }}\right\rangle$ abstracts a set $S$ of interval-guarded linear expressions, then for all $(B \Rightarrow F) \in S$, we have

$$
\left(B_{\text {int }} \Rightarrow F_{\text {inf }}\right) \preceq(B \Rightarrow F) \preceq\left(B_{\text {int }} \Rightarrow F_{\text {sup }}\right) \text {. }
$$

In fact, rather than tracking just a single interval-guarded bounding expression, an abstract state in our framework tracks bounded sets of such expressions. Using this abstraction, it is possible to approximate the semantics $\llbracket P \rrbracket$ of $P$ by two programs $P_{\text {sup }}$ and $P_{\text {inf }}$, whose semantics can be represented as a sum of a

1. Given a program $P$ for which an approximate smooth interpreter is to be constructed, use abstract interpretation to obtain programs $P_{\text {sup }}$ and $P_{\text {inf }}$ such that:

- $P_{\text {sup }}$ and $P_{\text {inf }}$ are interval-guarded linear programs.

$-\llbracket P_{\text {inf }} \rrbracket \preceq \llbracket P \rrbracket \preceq \llbracket P_{\text {sup }} \rrbracket \quad$ (here $\preceq$ is the pointwise ordering over functions).

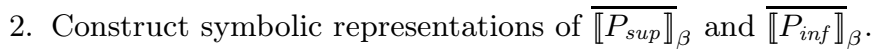

3. Let the approximate smooth interpreter for $P$ be a function that on any $\mathbf{x}$ and $\beta$, returns the Cartesian interval $\left[\overline{\llbracket P}_{\text {inf }} \rrbracket_{\beta}(\mathbf{x}), \overline{\llbracket P}_{P_{\text {sup }} \rrbracket}(\mathbf{x})\right]$.

Fig. 4. The approximate smooth interpretation algorithm 
bounded number of terms, each term being an interval-guarded linear expression. (We call such programs interval-guarded linear programs.)

The smoothed semantics of $P_{\text {sup }}$ and $P_{\text {inf }}$ can be computed in closed form, leading to an approximate smooth interpreter that is sketched in Fig. 4. In the rest of this section, we complete the above algorithm by describing the abstract interpreter in Step (1) and the analytic calculation in Step (2).

\section{Step 1: Abstraction Using Interval-Guarded Bounding Expressions}

Abstract domain. An abstract state $\sigma$ in our semantics is either a boundedsized set of interval-guarded bounding expressions (we let $N$ be a bound on the number of elements in such a set), or the special symbol $T$. The set of all abstract states is denoted by $\mathcal{A}$.

As usual, we define a partial order over the domain $\mathcal{A}$. Before defining this order, let us define a partial order $\unlhd$ over the universe $I G B$ that consists of all interval-guarded bounding expressions, as well as $\top$. For all $\sigma$, we have $\sigma \unlhd \top$. We also have:

$$
\begin{aligned}
\left\langle B, F_{\text {sup }}, F_{\text {inf }}\right\rangle & \unlhd\left\langle B^{\prime}, F_{\text {sup }}^{\prime}, F_{\text {inf }}^{\prime}\right\rangle \quad \text { iff } \quad B \Rightarrow B^{\prime} \text { and } \\
& \left(B^{\prime} \wedge B\right) \Rightarrow\left(F_{\text {sup }} \preceq F_{\text {sup }}^{\prime}\right) \wedge\left(F_{\text {inf }}^{\prime} \preceq F_{\text {inf }}\right) \text { and } \\
& \left(B^{\prime} \wedge \neg B\right) \Rightarrow\left(\mathbf{0} \preceq F_{\text {sup }}^{\prime}\right) \wedge\left(F_{\text {inf }}^{\prime} \preceq \mathbf{0}\right) .
\end{aligned}
$$

Intuitively, in the above, $B$ is a subinterval of $B^{\prime}$, and the expressions $\left(B^{\prime} \Rightarrow F_{\text {inf }}^{\prime}\right)$ and $\left(B^{\prime} \Rightarrow F_{\text {sup }}^{\prime}\right)$ define more relaxed bounds than $\left(B \Rightarrow F_{\text {inf }}\right)$ and $\left(B \Rightarrow F_{\text {sup }}\right)$.

Note that $\unlhd$ is not a lattice relation-e.g., the interval-guarded expressions $(1<x<2) \Rightarrow 1$ and $(3<x<4) \Rightarrow 5$ do not have a unique least upper bound. However, it is easy to give an algorithm $\sqcup_{I G B}$ that, given $H_{1}, H_{2} \in I G B$, returns a minimal, albeit nondeterministic, upper bound $H$ of $H_{1}$ and $H_{2}$ (i.e., $H_{1} \unlhd H$, $H_{2} \unlhd H$, and there is no $H^{\prime} \neq H$ such that $H^{\prime} \unlhd H, H_{1} \unlhd H^{\prime}$, and $H_{2} \unlhd H^{\prime}$ ).

Now can we define the partial order $\sqsubseteq$ over $\mathcal{A}$ that we use for abstract interpretation. For $\sigma_{1}, \sigma_{2} \in \mathcal{A}$, we have $\sigma_{1} \sqsubseteq \sigma_{2}$ iff either $\sigma_{2}=\top$, or if for all $H \in \sigma_{1}$, there exists $H^{\prime} \in \sigma_{2}$ such that $H \unlhd H^{\prime}$.

Once again, we can construct an algorithm $\sqcup_{\mathcal{A}}$ that, given $\sigma_{1}, \sigma_{2} \in \mathcal{A}$, returns a minimal upper bound $\sigma$ for $\sigma_{1}$ and $\sigma_{2}$. If $\sigma_{1}$ or $\sigma_{2}$ equals $T$, the algorithm simply returns $\top$. Otherwise, it executes the following program:

1. Let $\sigma^{\prime}:=\sigma_{1} \cup \sigma_{2}$.

2. While $\left|\sigma^{\prime}\right|>N$, repeatedly: (a) Nondeterministically select two elements $H_{1}, H_{2} \in \sigma^{\prime} ;(\mathrm{b})$ assign $\sigma^{\prime}:=\sigma^{\prime} \backslash\left\{H_{1}, H_{2}\right\} \cup\left(H_{1} \sqcup_{I G B} H_{2}\right)$;

3. Return $\sigma^{\prime}$.

Abstraction. The abstract semantics of the program $P$ is given by a map $\Psi_{P}^{\#}$ that associates an abstract state $\Psi_{P}^{\#}(u)$ with each node $u$ of $P$. To define this semantics, we need some more machinery. First, we need a way to capture the effect of an assignment node labeled by an expression $E$, on an abstract state $\sigma$. To this end, we define a notation $(E \circ \sigma)$ that denotes the composition of $E$ and $\sigma$. We have $E \circ \top=\top$ for all $E$. If $\sigma \neq \top$, then we 
have $(E \circ \sigma)=\left\{\left\langle B,\left(E \circ F_{\text {sup }}\right),\left(E \circ F_{\text {inf }}\right)\right\rangle:\left\langle B, F_{\text {sup }}, F_{\text {inf }}\right\rangle \in \sigma\right\}$. Applied to the abstract state $\sigma$, the assignment produces the abstract state $(E \circ \sigma)$.

Second, we must be able to propagate an abstract state $\sigma$ through a test node labeled by the boolean expression $C$. To achieve this, we define, for each abstract state $\sigma$ and boolean expression $C$, the composition $(C \circ \sigma)$ of $C$ and $\sigma$. In fact, we begin by defining the composition of $(C \circ H)$, where $H=\left\langle B, F_{\text {sup }}, F_{\text {inf }}\right\rangle$ is an interval-guarded boolean expression.

The idea here is that if a test node is labeled by $C$ and $H$ reaches the node, then $(C \circ H)$ is propagated along the true-branch. For simplicity, let us start with the case $B=$ true. Clearly, $(C \circ H)$ should be of the form $\left\langle B^{\prime}, F_{\text {sup }}, F_{\text {inf }}\right\rangle$ for some $B^{\prime}$. To see what $B^{\prime}$ should be, consider the scenario in Fig. 5, which shows the points $F_{\text {sup }}(\mathbf{x})$ and $F_{\text {inf }}(\mathbf{x})$ for a fixed $\mathbf{x} \in \mathbb{R}^{2}$. For all $F$ such that $F_{\text {inf }} \preceq F \preceq F_{\text {sup }}$, the point $F(\mathbf{x})$ must lie within the dashed box. So long as an "extreme

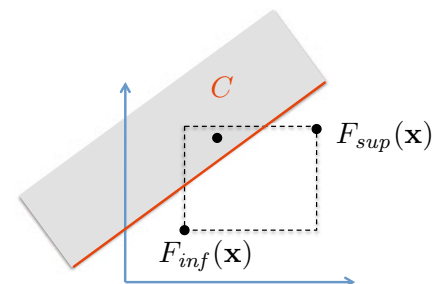

Fig. 5. Propagation through test $C$ point" of this box satisfies the constraint $C$ (the region to the left of the inclined line), $\mathbf{x}$ should satisfy $B^{\prime}$.

We need some more notation. For each function $F: \mathbb{R}^{k} \rightarrow \mathbb{R}^{k}$, let us define a collection of "components" $F^{1}, \ldots, F^{k}$ such that for all $i, F^{i}(\mathbf{x})=(F(\mathbf{x}))(i)$. A collection of component functions $F_{1}, \ldots, F_{k}: \mathbb{R}^{k} \rightarrow \mathbb{R}$ can be "combined" into a function $F=\left\langle F_{1}, \ldots, F_{k}\right\rangle: \mathbb{R}^{k} \rightarrow \mathbb{R}^{k}$, where for all $\mathbf{x}, F(\mathbf{x})=\left\langle F_{1}(\mathbf{x}), \ldots, F_{k}(\mathbf{x})\right\rangle$. The extreme points of our dashed box can now be seen to be obtained by taking all possible combinations of components from $F_{\text {sup }}$ and $F_{\text {inf }}$ and combining those functions. Then the property that some extreme point of the dashed box of Fig. [5] satisfies $C$ is captured by the formula

$$
\left(C \circ\left\langle F_{\text {sup }}^{1}, F_{\text {sup }}^{2}\right\rangle\right) \vee\left(C \circ\left\langle F_{\text {sup }}^{1}, F_{\text {inf }}^{2}\right\rangle\right) \vee\left(C \circ\left\langle F_{\text {inf }}^{1}, F_{\text {sup }}^{2}\right\rangle\right) \vee\left(C \circ\left\langle F_{\text {inf }}^{1}, F_{\text {inf }}^{2}\right\rangle\right) .
$$

The above can now be generalized to $k$-dimensions and the case $B \neq t r u e$. The composition of $C$ with an interval-guarded boolean expression $\left\langle B, F_{\text {sup }}, F_{\text {inf }}\right\rangle$ is defined to be $C \circ\left\langle B, F_{\text {sup }}, F_{\text {inf }}\right\rangle=\left\langle B^{\prime}, F_{\text {sup }}, F_{\text {inf }}\right\rangle$, where

$$
B^{\prime}=B \wedge\left(\bigvee_{G^{i} \in\left\{F_{\text {sup }}^{i}, F_{\text {inf }}^{i}\right\}}\left(C \circ\left\langle G^{1}, \ldots, G^{k}\right\rangle\right)\right) .
$$

Let us now lift this composition operator to abstract states. We define $C$ 。 $\top=\top$ for all $C$. For all $\sigma \neq \top$, we have $(C \circ \sigma)=\left\{C \circ\left\langle B, F_{\text {sup }}, F_{\text {inf }}\right\rangle\right.$ : $\left.\left\langle B, F_{\text {sup }}, F_{\text {inf }}\right\rangle \in \sigma\right\}$. Finally, for any boolean expression $C$, let us define $C^{\#}$ to be an interval that overapproximates $C$.

The abstract semantics $\Psi_{P}^{\#}$ of $P$ is now defined using the algorithm in Figure 6. We note that $\Psi_{P}^{\#}$ can have different values depending on the sequence of nondeterministic choices made by our upper-bound operators. However, every resolution of such choices leads to an abstract semantics that can support a sound and robust approximate smooth interpreter. Consequently, from now on, 
1. If $u$ is the entry node of $P$ and its outgoing edge leads to $v$, then assign $\Psi_{P}^{\#}(v):=$ $\{\langle$ true, $\mathbf{x}, \mathbf{x}\rangle\}$. Assign $\Psi_{P}^{\#}\left(v^{\prime}\right)=\emptyset$ for every other node $v^{\prime}$.

2. Until fixpoint, repeat:

(a) If $u$ is an assignment node labeled by $E$ and its outgoing edge leads to $v$, then $\operatorname{assign} \Psi_{P}^{\#}(v):=\Psi_{P}^{\#}(v) \sqcup\left(E \circ \Psi_{P}^{\#}(u)\right)$.

(b) Suppose $u$ is a branch node; then let $v_{t}$ and $v_{f}$ respectively be the targets of the true- and false-edges out of it, and let $Q_{t}=\operatorname{Test}(u)$ and $Q_{f}=\neg T e s t(u)$

$$
\begin{aligned}
& \Psi_{P}^{\#}\left(v_{t}\right):=\Psi_{P}^{\#}\left(v_{t}\right) \sqcup\left\{Q_{t} \circ\left\langle B, F_{\text {sup }}, F_{\text {inf }}\right\rangle:\left\langle B, F_{\text {sup }}, F_{\text {inf }}\right\rangle \in \Psi_{P}^{\#}(u)\right\} \\
& \Psi_{P}^{\#}\left(v_{f}\right):=\Psi_{P}^{\#}\left(v_{f}\right) \sqcup\left\{Q_{f} \circ\left\langle B, F_{\text {sup }}, F_{\text {inf }}\right\rangle:\left\langle B, F_{\text {sup }}, F_{\text {inf }}\right\rangle \in \Psi_{P}^{\#}(u)\right\}
\end{aligned}
$$

(c) If $u$ is a junction node with an out-edge to $v$, then $\Psi_{P}^{\#}(v):=\Psi_{P}^{\#}(u) \sqcup \Psi_{P}^{\#}(v)$.

Fig. 6. Algorithm to compute $\Psi_{P}^{\#}$

we will ignore the fact that $\Psi_{P}^{\#}$ actually represents a family of maps, and instead view it as a function of $P$.

Widening. As our abstract domain is infinite, our fixpoint computation does not guarantee termination. For termination of abstract interpretation, our domain needs a widening operator $[8$. For example, one such operator $\nabla$ can be defined as follows.

First we define $\nabla$ on interval-guarded bounding expressions. Let us suppose $\left\langle B_{w}, F_{\text {sup }}^{\prime \prime}, F_{\text {inf }}^{\prime \prime}\right\rangle=\left\langle B, F_{\text {sup }}, F_{\text {inf }}\right\rangle \nabla\left\langle B^{\prime}, F_{\text {sup }}^{\prime}, F_{\text {inf }}^{\prime}\right\rangle$. Then we have:

- $B_{w}=B \nabla_{\text {int }} B^{\prime}$, where $\nabla_{\text {int }}$ is the standard widening operator for the interval domain [8].

- $F_{\text {sup }}^{\prime \prime}$ is a minimal function in the pointwise order $\preceq$ such that for all $\mathbf{x} \in B_{w}$, we have $F_{\text {sup }}^{\prime \prime}(\mathbf{x}) \geq\left(B \Rightarrow F_{\text {sup }}\right)(\mathbf{x})$ and $F_{\text {sup }}^{\prime \prime}(\mathbf{x}) \geq\left(B^{\prime} \Rightarrow F_{\text {sup }}^{\prime}\right)(\mathbf{x})$.

- $F_{\text {inf }}^{\prime \prime}$ is a maximal function such that for all $\mathbf{x} \in B_{w}$, we have $F_{\text {sup }}^{\prime \prime}(\mathbf{x}) \leq$ $\left(B \Rightarrow F_{\text {inf }}\right)(\mathbf{x})$ and $F_{\text {inf }}^{\prime \prime}(\mathbf{x}) \leq\left(B^{\prime} \Rightarrow F_{\text {inf }}^{\prime}\right)(\mathbf{x})$.

This operator is now lifted to abstract states in the natural way.

Computing $P_{\text {sup }}$ and $P_{\text {inf }}$. Now we can compute the interval-guarded linear programs $P_{\text {sup }}$ and $P_{\text {inf }}$ that bound $P$. Let ex be the exit node of $P$, and let $\Psi_{P}^{\#}(e x)=\left\{\left\langle B^{1}, F_{\text {sup }}^{1}, F_{\text {inf }}^{1}\right\rangle, \ldots,\left\langle B^{n}, F_{\text {sup }}^{n}, F_{\text {inf }}^{n}\right\rangle\right\}$ for some $n \leq N$. Then, the symbolic representation of the semantics $\llbracket P_{\text {sup }} \rrbracket$ and $\llbracket P_{\text {inf }} \rrbracket$ of $P_{\text {sup }}$ and $P_{\text {inf }}$ is as follows:

$$
\llbracket P_{\text {sup }} \rrbracket=\sum_{i}^{N} \llbracket B^{i} \Rightarrow F_{\text {sup }}^{i} \rrbracket \quad \llbracket P_{\text {inf }} \rrbracket=\sum_{i}^{N} \llbracket B^{i} \Rightarrow F_{\text {inf }}^{i} \rrbracket .
$$

\section{Step 2: Symbolic Convolution of Interval-Guarded Linear Programs}

Now we give a closed-form expression for the smoothed semantics of $P_{\text {sup }}$ (the case of $\llbracket P_{\text {inf }} \rrbracket$ is symmetric). We have:

$$
\overline{\llbracket P_{\text {sup }} \rrbracket}(\mathbf{x})=\int_{\mathbf{r} \in \mathbb{R}^{k}} \llbracket P_{\text {sup }} \rrbracket(\mathbf{r}) \mathcal{N}(\mathbf{x}-\mathbf{r}) d \mathbf{r}=\sum_{i} \int_{\mathbf{r} \in \mathbb{R}^{k}} \llbracket B^{i} \Rightarrow F_{\text {sup }}^{i} \rrbracket(\mathbf{r}) \mathcal{N}(\mathbf{x}-\mathbf{r}) d \mathbf{r} .
$$


To solve this integral, we first observe that by our assumptions, $\mathcal{N}$ is the joint distribution of $k$ univariate independent Gaussians with the same standard deviation $\beta$. Therefore, we can split $\mathcal{N}$ into a product of univariate Gaussians $\mathcal{N}_{1}, \ldots, \mathcal{N}_{k}$, where the Gaussian $\mathcal{N}_{j}$-th ranges over $x_{j}$. Letting $r_{j}$ be the $j$-th component of $\mathbf{r}$, we have:

$$
\begin{aligned}
\int_{\mathbf{r} \in \mathbb{R}^{k}} \llbracket B^{i} \Rightarrow F_{\text {sup }}^{i} \rrbracket(\mathbf{r}) \mathcal{N}(\mathbf{x}-\mathbf{r}) d \mathbf{r}= \\
\quad \int_{-\infty}^{\infty} \ldots\left(\int_{-\infty}^{\infty} \llbracket B^{i} \Rightarrow F_{\text {sup }}^{i} \rrbracket(\mathbf{r}) \mathcal{N}_{1}\left(x_{1}-r_{1}\right) d r_{1}\right) \ldots \mathcal{N}_{k}\left(x_{k}-r_{k}\right) d r_{k} .
\end{aligned}
$$

Thus, due to independence of the $x_{j}$ 's, it is possible to reduce the vector integral involved in convolution to a sequence of integrals over one variable. Each of these integrals will be of the form $\int_{-\infty}^{\infty} \llbracket B^{i} \Rightarrow F_{\text {sup }}^{i} \rrbracket(\mathbf{r}) \mathcal{N}_{j}\left(x_{j}-r_{j}\right) d r_{j}$. Projecting the interval $B^{i}$ over the axis for $x_{j}$ leaves us with an integral like the one we solved analytically in Example 1. This allows us to represent the result of smooth interpretation as a finite sum of closed form expressions.

\section{Properties of the Interpreter}

Now we prove that the algorithm as defined above actually satisfies the properties claimed in Sec. 3. We first establish that the approximate smooth interpreter presented in this paper is sound. Next we show that under a weak assumption about $\beta$, it is robust and $\beta$-robust as well.

Theorem 1. The approximate smooth interpreter of Figure 4 is sound.

Proof: In order to prove soundness, we need to prove that ${\overline{\llbracket P \rrbracket_{\beta}}}_{\beta}(\mathbf{x}) \in$ Smooth $_{P}$ $(\mathbf{x}, \beta)$. This follows directly from the soundness of abstract interpretation thanks to a property of Gaussian convolution: that it is a monotone map on the pointwise partial order $\preceq$ of functions between $\mathbb{R}^{k}$.

First, we have seen how to use abstract interpretation to produce two programs $P_{\text {inf }}$ and $P_{\text {sup }}$ such that $\llbracket P_{\text {inf }} \rrbracket \preceq \llbracket P \rrbracket \preceq \llbracket P_{\text {sup }} \rrbracket$.

Now, we defined $\operatorname{Smooth}_{P}(\mathbf{x}, \beta)$ as the interval $\left[\overline{\llbracket P_{\text {inf }} \rrbracket}(\mathbf{x}), \overline{\llbracket P_{\text {sup }} \rrbracket}(\mathbf{x})\right]$; therefore, all we have to do to prove soundness is to show that $\overline{\llbracket P_{\text {inf }} \rrbracket} \preceq \overline{\llbracket P \rrbracket_{\beta}} \preceq \overline{\llbracket P_{\text {sup }} \rrbracket}$. In other words, we need to show that Gaussian smoothing preserves the ordering among functions.

This follows directly from a property of convolution. Let $F \preceq G$ for functions $F, G: \mathbb{R}^{k} \rightarrow \mathbb{R}^{k}$, and $H: \mathbb{R}^{k} \rightarrow \mathbb{R}^{k}$ be any function satisfying $H(\mathbf{x})>0$ for all $\mathbf{x}$ (note that the Gaussian function satisfies this property). Also, let $F_{H}$ and $G_{H}$ be respectively the convolutions of $F$ and $H$, and $G$ and $H$. Then we have $F^{\prime} \preceq H^{\prime}$.

Theorem 2. For every constant $\epsilon>0$, the approximate smooth interpreter of Figure 4 is robust in the region $\beta>\epsilon$. 
Proof: To prove robustness of $\operatorname{Smooth}_{P}$, it suffices to show that both $\overline{\llbracket P_{\text {inf }} \rrbracket}$ and $\overline{\llbracket P_{\text {sup }} \rrbracket}$ satisfy the robustness condition. We focus on proving $\overline{\llbracket P_{\text {sup }} \rrbracket}$ robust, since the proof for $\overline{\llbracket P_{\text {inf }} \rrbracket}$ is symmetric. Now, we know that $\llbracket P_{\text {sup }} \rrbracket$ has the form $\llbracket P_{\text {sup }} \rrbracket(\mathbf{x})=\sum_{i=0}^{N} B_{i}(\mathbf{x}) F_{i}(\mathbf{x})$, where $B_{i}(\mathbf{x})$ is an interval in $\mathbb{R}^{k}$ and $F_{i}(\mathbf{x})$ is a linear function. Hence we have:

$$
\llbracket \overline{P_{\text {sup }}} \rrbracket(\mathbf{x})=\int_{\mathbf{r} \in \mathbb{R}^{k}}\left(\sum_{i=0}^{N} B_{i}(\mathbf{r}) \cdot F_{i}(\mathbf{r})\right) \cdot \mathcal{N}(\mathbf{r}-\mathbf{x}, \beta) d \mathbf{r} .
$$

where $\mathcal{N}$ is the joint density function of $k$ independent 1-D Gaussians. It is easy to see that this function is differentiable arbitrarily many times in $x_{j}$. We have:

$$
\frac{\partial \llbracket \overline{P_{\text {sup }}} \rrbracket(\mathbf{x})}{\partial x_{j}}=\int_{\mathbf{r} \in \mathbb{R}^{k}}\left(\sum_{i=0}^{N} B_{i}(\mathbf{r}) \cdot F_{i}(\mathbf{r})\right) \cdot\left(\frac{\partial \mathcal{N}(\mathbf{r}-\mathbf{x}, \beta)}{\partial x_{j}}\right) d \mathbf{r}
$$

Now note that $\frac{\partial \mathcal{N}(\mathbf{r}-\mathbf{x}, \beta)}{\partial x_{j}}=\frac{\left(r_{j}-x_{j}\right)}{\beta^{2}} \cdot \mathcal{N}(\mathbf{r}-\mathbf{x}, \beta)$. Hence,

$$
\begin{aligned}
\frac{\partial \llbracket \overline{P_{\text {sup }} \rrbracket}}{\partial x_{j}}(\mathbf{x}) & =\int_{\mathbf{r} \in \mathbb{R}^{k}}\left(\frac{1}{\beta^{2}} \sum_{i=0}^{N} B_{i}(\mathbf{r}) \cdot F_{i}(\mathbf{r}) \cdot\left(r_{j}-x_{j}\right)\right) \cdot \mathcal{N}(\mathbf{r}-\mathbf{x}, \beta) d \mathbf{r} \\
& =\left(\frac{1}{\beta^{2}} \sum_{i=0}^{N} \int_{\mathbf{r} \in B_{i}} F_{i}(\mathbf{r}) \cdot\left(r_{j}-x_{j}\right) \cdot \mathcal{N}(\mathbf{r}-\mathbf{x}, \beta) d \mathbf{r}\right.
\end{aligned}
$$

Each $F_{i}$ is a linear function of type $\mathbb{R}^{k} \rightarrow \mathbb{R}^{k}$, so for each $n<k$, we have $\left(F_{i}(\mathbf{r})\right)(n)=\left(\sum_{l=0}^{k-1} \alpha_{i, l, n} \cdot r_{l}\right)+\gamma_{i, n}$ for constants $\alpha_{i, j, n}$ and $\gamma_{i, n}$. Substituting in Eqn. 2, we find that the $n$-th coordinate of the vector-valued expression $\frac{\partial \llbracket \overline{P_{\text {sup }}} \rrbracket(\mathbf{x})}{\partial x_{j}}$ can be expanded into a linear sum of terms as below:

$$
\begin{array}{r}
\frac{1}{\beta^{2}} \int_{r_{j}=a}^{b} \mathcal{N}\left(r_{j}-x_{j}, \beta\right) d r_{j} \\
\frac{1}{\beta^{2}} \int_{r_{j}=a}^{b}\left(r_{j}-x_{j}\right) \cdot \mathcal{N}\left(r_{j}-x_{j}, \beta\right) d r_{j} \\
\frac{1}{\beta^{2}} \int_{r_{j}=a}^{b} r_{j} \cdot \mathcal{N}\left(r_{j}-x_{j}, \beta\right) d r_{j} \\
\frac{1}{\beta^{2}} \int_{r_{j}=a}^{b} r_{j} \cdot\left(r_{j}-x_{j}\right) \cdot \mathcal{N}\left(r_{j}-x_{j}, \beta\right) d r_{j}
\end{array}
$$

In order to show that $\left|\frac{\partial \llbracket \overline{P_{s u p}} \rrbracket(\mathbf{x})}{\partial x_{j}}\right|$ is bounded by a linear function, we first observe that for all $\beta>\epsilon$, the multiplier $\frac{1}{\beta^{2}}$ is bounded by a constant. Now we show that the each integrals in the above is bounded by a function that is linear in $x_{j}$, even when $a=-\infty$ or $b=\infty$.

It is easy to see that of these integrals, the first two are bounded in value for any value of $x_{j}$; this follows from the fact that $\mathcal{N}$ decays exponentially as its first 
argument goes to infinity. The last integral will also be bounded as a function of $x_{j}$, while the second to last function can grow linearly with $x_{j}$. It follows that $\left|\frac{\partial \llbracket \overline{P_{\text {sup }}} \rrbracket(\mathbf{x})}{\partial x_{j}}\right|$ can grow only linearly with $\mathbf{x}$ and $\beta$. Hence $\overline{\llbracket P_{\text {sup }} \rrbracket}$ is robust.

Theorem 3. For every constant $\epsilon>0$, the approximate smooth interpreter from Figure 4 is $\beta$-robust in the region $\beta>\epsilon$.

Proof: As in the proof of Theorem 2] it suffices to only consider the derivative of $P_{\text {sup }}$ (w.r.t. $\beta$ this time) - the case for $P_{\text {inf }}$ is symmetric. From the definition of smoothing, we have:

$$
\begin{aligned}
\frac{\partial \llbracket \overline{P_{\text {sup }} \rrbracket}}{\partial \beta} & =\int_{\mathbf{r} \in \mathbb{R}^{k}}\left(\sum_{i=0}^{N} B_{i}(\mathbf{r}) \cdot F_{i}(\mathbf{r})\right) \cdot \frac{\partial \mathcal{N}(\mathbf{r}-\mathbf{x}, \beta)}{\partial \beta} d \mathbf{r} \\
& \left.=\int_{\mathbf{r} \in \mathbb{R}^{k}}\left(\sum_{i=0}^{N} B_{i}(\mathbf{r}) \cdot F_{i}(\mathbf{r})\right) \cdot\left(\frac{\|\mathbf{r}-\mathbf{x}\|^{2}}{\beta^{3}}-\frac{k}{\beta}\right)\right) \cdot \mathcal{N}(\mathbf{r}-\mathbf{x}, \beta) d \mathbf{r} \\
& =\sum_{i=0}^{N} \int_{\mathbf{r} \in B_{i}} F_{i}(\mathbf{r}) \cdot\left(\frac{\|\mathbf{r}-\mathbf{x}\|^{2}}{\beta^{3}}-\frac{k}{\beta}\right) \cdot \mathcal{N}(\mathbf{r}-\mathbf{x}, \beta) d \mathbf{r} .
\end{aligned}
$$

Just like in the proof of robustness, we can decompose the integral into a linear sum of terms of one of the following forms:

$$
\begin{array}{r}
\frac{1}{\beta^{3}} \int_{r_{j}=a}^{b}\left(r_{j}-x_{j}\right)^{2} \cdot \mathcal{N}\left(r_{j}-x_{j}, \beta\right) d r_{j} \\
\frac{1}{\beta^{3}} \int_{r_{j}=a}^{b} r_{j} \cdot\left(r_{j}-x_{j}\right)^{2} \cdot \mathcal{N}\left(r_{j}-x_{j}, \beta\right) d r_{j} \\
\frac{1}{\beta} \int_{r_{j}=a}^{b} r_{j} \cdot \mathcal{N}\left(r_{j}-x_{j}, \beta\right) d r_{j} \\
\frac{1}{\beta} \int_{r_{j}=a}^{b} \mathcal{N}\left(r_{j}-x_{j}, \beta\right) d r_{j}
\end{array}
$$

The first and last terms are clearly bounded by constants. The third term is similar to the term we saw in the proof of robustness, and is bounded by a linear function; in fact, when $a$ and $b$ go to infinity, the term corresponds to the mean of a Gaussian centered at $x$. As for the second term, it is bounded when $a$ and $b$ are bounded, but will grow as $O\left(x_{j}\right)$ when $a$ or $b$ are $-\infty$ or $\infty$ respectively. Putting the facts together, we note that $\left|\frac{\partial \llbracket \overline{P_{\text {sup }}} \rrbracket(\mathbf{x})}{\partial \beta}\right|$ is bounded by a linear function of $\mathbf{x}$ and $\beta$, which completes the proof of $\beta$-robustness.

\section{Related Work and Conclusion}

In a recent paper [4], we introduced smooth interpretation as a program approximation that facilitates more effective use of numerical optimization in reasoning 
about programs. While the paper showed the effectiveness of the method, it left open a lot of theoretical questions. For example, while we implemented and empirically evaluated a smooth interpreter, could we also formally characterize smooth interpretation? How did program smoothing relate to program abstraction? In the present paper, we have answered the above questions.

Regarding related work, Gaussian smoothing is ubiquitous in signal and image processing [12]. Also, the idea of using smooth, robust approximations to enable optimization of non-smooth functions has been previously studied in the optimization community 11. However, these approaches are technically very different from ours, and we were the first to propose and find an application for Gaussian smoothing of programs written in a general-purpose programming language. As for work in the software engineering community, aside from a cursory note by DeMillo and Lipton [9, there does not seem to be any prior proposal here for the use of "smooth" models of programs (although some recent work in program verification studies continuity properties [23] of programs).

The abstract interpretation used in our smoothing framework is closely related to a large body of prior work on static analysis, in particular analysis using intervals [8], interval equalities [6], and interval polyhedra [5]. However, so far as we know, there is no existing abstract domain that can conditionally bound the denotational semantics of a program from above and below.

\section{References}

1. Burnim, J., Juvekar, S., Sen, K.: Wise: Automated test generation for worst-case complexity. In: ICSE, pp. 463-473 (2009)

2. Chaudhuri, S., Gulwani, S., Lublinerman, R.: Continuity analysis of programs. In: POPL (2010)

3. Chaudhuri, S., Gulwani, S., Lublinerman, R., Navidpour, S.: Proving programs robust (2011)

4. Chaudhuri, S., Solar-Lezama, A.: Smooth interpretation. In: PLDI (2010)

5. Chen, L., Miné, A., Wang, J., Cousot, P.: Interval polyhedra: An abstract domain to infer interval linear relationships. In: Palsberg, J., Su, Z. (eds.) SAS 2009. LNCS, vol. 5673, pp. 309-325. Springer, Heidelberg (2009)

6. Chen, L., Miné, A., Wang, J., Cousot, P.: An abstract domain to discover interval linear equalities. In: Barthe, G., Hermenegildo, M. (eds.) VMCAI 2010. LNCS, vol. 5944, pp. 112-128. Springer, Heidelberg (2010)

7. Chen, X.: Convergence of the BFGS method for LC convex constrained optimization. SIAM J. Control and Optim. 14, 2063 (1996)

8. Cousot, P., Cousot, R.: Abstract interpretation: a unified lattice model for static analysis of programs by construction or approximation of fixpoints. In: POPL (1977)

9. DeMillo, R., Lipton, R.: Defining software by continuous, smooth functions. IEEE Transactions on Software Engineering 17(4), 383-384 (1991)

10. Hager, W.W., Zhang, H.: A survey of nonlinear conjugate gradient methods. Pacific Journal of Optimization 2(1), 35-58 (2006)

11. Nesterov, Y.: Smooth minimization of non-smooth functions. Mathematical Programming 103(1), 127-152 (2005)

12. Russ, J.: The image processing handbook. CRC Press, Boca Raton (2007) 